\title{
Periodic Nano-Grating Structures Produced by Femtosecond Laser Pulses for Metals with Low- and High-Melting Points
}

\author{
Masaki HASHIDA $^{1,2}$, Yasuhiro MIYASAKA ${ }^{1,2}$, Yoshinobu IKUTA ${ }^{1,2}$, Kazuto OTANI ${ }^{1,2}$, \\ Shigeki TOKITA ${ }^{1,2}$ and Shuji SAKABE ${ }^{1,2}$ \\ ${ }^{1}$ Advanced Research Center for Beam Science, Institute for Chemical Research, Kyoto University, \\ Gokasho, Uji, Kyoto 611-0011, Japan \\ E-mail: hashida@laser.kuicr.kyoto-u.ac.jp \\ ${ }^{2}$ Department of Physics, Graduate School of Science, Kyoto University, Kitashirakawa, Sakyo, \\ Kyoto 606-8502, Japan
}

\begin{abstract}
Periodic nano-grating structures self-formed on the surface of several metals by femtosecond laser pulses are analyzed by two-dimensional Fourier-transform. For the self-formation of grating structures on metal surfaces, the period of the structures depend on laser fluence. This dependence is the same for all metals, although the range of laser fluence in which the structures are formed differs between metals. For Ti with relatively low melting temperature $\left(T_{\mathrm{m}}=1938 \mathrm{~K}\right)$ the grating nanostructures were self-formed for laser fluence of $0.09-0.45 \mathrm{~J} / \mathrm{cm}^{2}$, while for Mo with high melting temperature $\left(T_{\mathrm{m}}=2888 \mathrm{~K}\right)$ structures were formed for laser fluence of $0.18-1.1 \mathrm{~J} / \mathrm{cm}^{2}$. Higher the melting temperature, upper limit of grating structure formation tends to be higher. These experimental results indicate that the period of the self-formed grating structure depend not on metal characteristics, but rather on the density of the surface plasma produced by a laser pulse.
\end{abstract}

DOI:10.2961/jlmn.2012.02.0011

Keywords: Periodic grating structures, Metals, Femtosecond laser, Melting points, Parametric decay

\section{Introduction}

Recently, the formation of grating structures on metal surfaces has been observed[1]-[23]. For fluence levels near the low ablation threshold, the grating structures had a period of $300 \mathrm{~nm}$, which was much shorter than the laser wavelength of $800 \mathrm{~nm}$. The formation of grating period, especially for $\mathrm{Cu}$ with a $100 \mathrm{fs}$ laser pulse, depends on laser fluence [24], and this phenomenon is well explained by the parametric decay model [25] proposed by Sakabe et al. We have measured the dependence on laser fluence of the grating period in $\mathrm{Ti}, \mathrm{Pt}, \mathrm{Mo}$, and $\mathrm{W}[1]$ and found that the experimental results agreed reasonably well with this model.

In parametric decay model, a femtosecond laser pulse decays into a surface-plasma wave and a scattered electromagnetic wave in which wavelength is longer than that of incident laser. Angular distribution of scattered wave was calculated and reported [26]. The plasma wave produces an ion-enriched local area on the surface. These ions experience a strong Coulomb repulsive force and can be ejected into a vacuum: a Coulomb explosion[27][28] occurs. Through this process, periodic grating structures are formed. This mechanism may also be responsible for the creation of amorphous metals. The relation between the formation of a surface grating structure and its crystallization has been investigated. It was found that the periodic grating structure with amorphous state on copper thin film was produced at the appropriate laser fluence[29]. When periodic nanostructure on metal with amorphous state can be produced, thin metals with new functionality for electrical, optical, and thermal properties are expected.

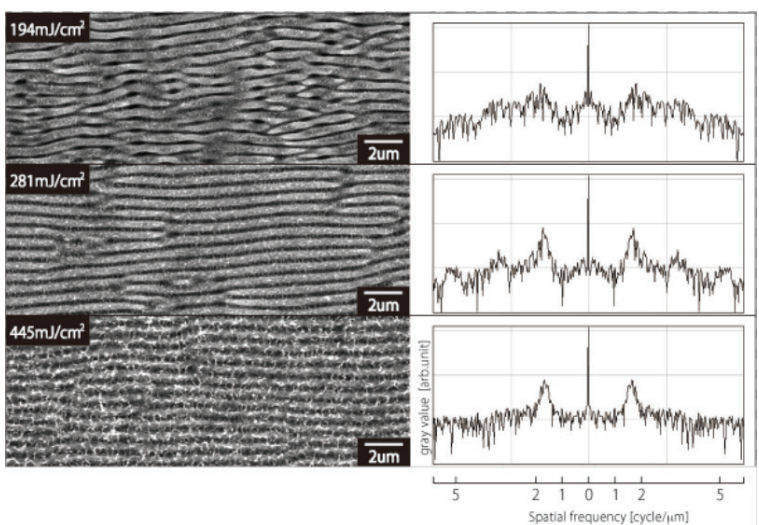

Fig. 1 SEM images of periodic nano-grating structures on tungsten and the Fourier transform spectra after 200 laser pulses irradiation at different laser fluences of (a) $194 \mathrm{~mJ} / \mathrm{cm}^{2}$, (b) $281 \mathrm{~mJ} / \mathrm{cm}^{2}$, (c) $445 \mathrm{~mJ} / \mathrm{cm}^{2}$.

However the relation between the type of metal and the period of grating structures with different number of pulse irradiations has not been investigated, and therefore the amount of systematic experimental data is insufficient for discussing the mechanism at present. The generation of the surface plasma wave leads to formation of periodic structures is not yet fully understood. Several mechanisms of nanostructure formation for materials are proposed [30][33], however we need further investigation. In this paper, we investigated by electron microscopy the state of surfaces irradiated with femtosecond laser pulses for Ti, Pt, Mo, and $\mathrm{W}$ metals. In addition, we evaluated the dependence of 
grating structure period on laser fluence to discuss physical property (melting temperature $T_{\mathrm{m}}$ ) of metals and the validity of the parametric decay model.

\section{Experiments}

In the experiments, $\mathrm{T}^{6}$-laser system $(\lambda=800-\mathrm{nm}, \tau=160$ fs, $10-\mathrm{Hz}$ ) was used[34]. The laser beam was focused to a spot size of $45 \mu \mathrm{m} \phi$ on the target surface with a lens ( $f=$ $10 \mathrm{~cm}$ ), at normal incidence in air. To avoid non-uniformity of structure in the irradiated area on the surface, the laser intensity distribution was adjusted to be spatially uniform. The targets were Ti, Pt, Mo, and W metals, which had been mechanically polished. The roughness, Ra, was less than 2 $\mathrm{nm}$ for all metals. The energy was varied in the range of 1.1-34 mJ, corresponding to fluence of $F=50-2100$ $\mathrm{mJ} / \mathrm{cm}^{2}$. The number of irradiating pulses was 200 in all experiments. Laser-produced surface structures were examined by scanning electron microscopy (JSM-5560, JEOL). The period of grating structure was determined by reading the peak value in the frequency domain after taking the Fourier transform for the $20 \mu \mathrm{m} \times 15 \mu \mathrm{m}$ area of the SEM image, which is equivalent to the laser irradiated area on the targets. The resolution of the present measurements of the grating period was better than $34 \mathrm{~nm}$. The period of the grating structure was obtained by analyzing a set of $10 \mathrm{ir}-$ radiated spots on a metal surface. Figure 1 shows typical SEM images and the corresponding Fourier transform spectra. To evaluate the ablation rate, the crater produced by 200 laser pulses was measured by confocal laser scanning microscopy (HL-150, Lasertec).

\section{Results and discussions}

Figure 2 shows the dependence of the grating structure period on laser fluence for $\mathrm{Ti}, \mathrm{Pt}, \mathrm{Mo}$, and $\mathrm{W}$ metals. The upper limit on the laser fluence at which the periodic structures are formed can be seen; let us define this fluence as $F_{\mathrm{M}}$. The laser fluence in Fig. 2 is normalized by the fluence $F_{\mathrm{M}}$ (see Table 1 for $F_{\mathrm{M}}$ of each metal). As the laser fluence increases, the period increases up to about $700 \mathrm{~nm}$ for all metals, and this maximum period is obtained at the fluence $F_{\mathrm{M}}$.

Let us compare these experimental results with the predictions from the model of plasma wave induction by parametric decay of laser light [28]. Here, the model is briefly described. The parametric process of photon $\rightarrow$ photon + plasmon can occur on a plasma surface as well as in a bulk plasma (i.e., stimulated Raman scattering). The parametric conditions of $\omega_{\mathrm{L}}=\omega_{2}+\omega_{\mathrm{SP}}$ and $\mathrm{k}_{\mathrm{L}}=\mathrm{k}_{2}+\mathrm{k}_{\mathrm{SP}}$, where the subscripts L, 2, and SP indicate the incident laser light, scattered light, and surface plasma wave, respectively, are reduced to

$$
\begin{aligned}
& \omega_{\mathrm{L}}-\omega_{\mathrm{SP}}=c k_{\mathrm{SP}}-c k_{\mathrm{L}}, \omega_{\mathrm{L}}=c k_{\mathrm{L}}, \\
& \omega_{\mathrm{SP}}{ }^{2}=c^{2}{k_{\mathrm{SP}}}^{2}+\frac{1}{2} \omega_{\mathrm{p}}{ }^{2}-\left(c^{4}{k_{\mathrm{SP}}}^{4}+\frac{1}{4}{\omega_{\mathrm{p}}}^{2}\right)^{1 / 2} .
\end{aligned}
$$

The wavenumber of the plasma wave induced by the parametric process can be related to the plasma frequency, and the $k_{\mathrm{L}} / k_{\mathrm{SP}}$ ratio $\left(=\lambda_{\mathrm{SP}} / \lambda_{\mathrm{L}} ; \lambda\right.$ is the wavelength) changes from 0.5 to 0.85 for plasma frequencies in the range of $0<$ $\omega_{\mathrm{p}} / 2^{0.5}<\omega_{\mathrm{L}}$, where the plasma wavenumber increases as the plasma frequency decreases.
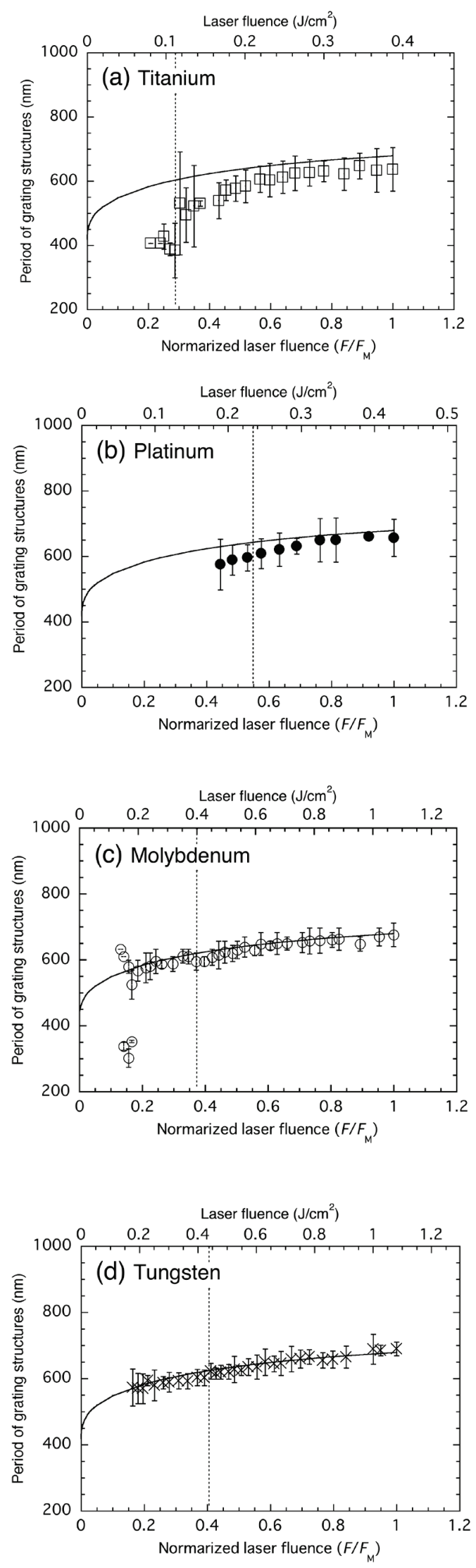

Fig. 2 Laser fluence dependence of the grating structures period for (a) Ti, (b) Pt, (c)Mo, and (d) W. Solid lines show calculation results according to the parametric decay model. 


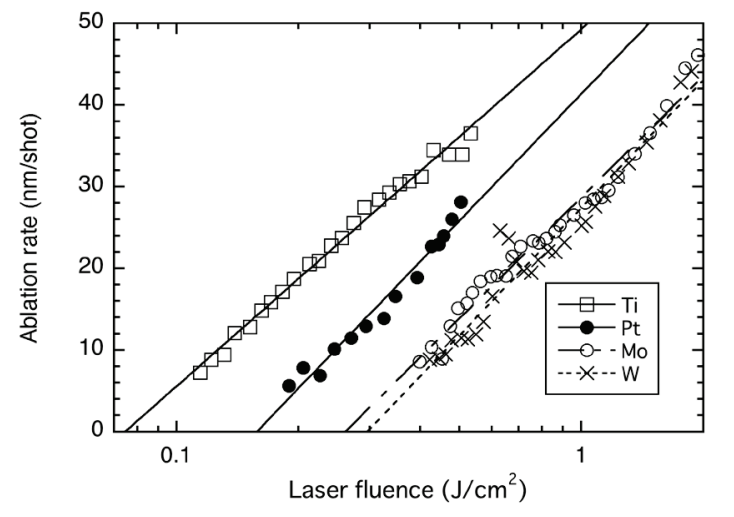

Fig. 3 Laser fluence dependence of ablation rate for Ti, Pt, Mo, and W. Experimental data were fit to well known logarithmic function.

As mentioned above, assuming that the self-formation is induced by the plasma wave, the grating period correspond to the wavelength of the induced plasma wave, and the fluence dependence of the period can be reduced to plasma density dependence. The dependence of the surface electron density $n_{\mathrm{es}}$ on the laser fluence $F_{\mathrm{L}}$ can be interpreted as follows. The electron density $n_{\mathrm{e}}$ of the plasma bulk produced on the surface by the laser is proportional to the laser energy: $n_{\mathrm{e}} \propto F_{\mathrm{L}}$. The heated plasma bulk with temperature $T_{\mathrm{e}}$ expands at the sonic speed $c_{\mathrm{s}}=\left(k_{\mathrm{B}} T_{\mathrm{e}} / m\right)^{0.5}$, and the surface electron density decreases from the bulk density as $n_{\mathrm{e}} / c_{\mathrm{s}}$, and the temperature is proportional to the laser energy: $T_{\mathrm{e}} \propto F_{\mathrm{L}}$. Therefore, the surface electron density is related to the laser fluence as $n_{\mathrm{es}} \propto n_{\mathrm{e}} / c_{\mathrm{s}} \propto n_{\mathrm{e}} / T^{1 / 2} \propto F_{\mathrm{L}}^{1 / 2}$. It is reasonable to assume that the plasma frequency is $\omega_{\mathrm{p}}=$ $2^{0.5} \omega_{\mathrm{L}}$ for the laser fluence $F_{\mathrm{M}}$ since no grating structures are produced at laser fluence over $F_{\mathrm{M}}$. In this case, $n_{\mathrm{es}}\left[\mathrm{cm}^{-}\right.$ $\left.{ }^{3}\right]=3.5 \times 10^{21}\left(F_{\mathrm{L}} / F_{\mathrm{M}}\right)^{1 / 2}$. Applying this expression together with $\omega_{\mathrm{p}}=\left(4 \pi n_{\mathrm{es}} e^{2} / m\right)^{0.5}$ to the dependence of $\lambda_{\mathrm{SP}} / \lambda_{\mathrm{L}}$ on $\omega_{\mathrm{p}}$, the spatial dependence of the laser fluence is obtained. This relation is shown as a solid line in Fig. 2. For each metal, the experimental results agree reasonably well with this model, although there is disagreement in the relatively low fluence range. This disagreement is considered to arise from non-uniformity of ablation. At laser fluence near the ablation threshold, the amount of ablated plasma is too small to construct a uniform plasma surface. The measured ablation rate is shown in Fig. 3. The threshold of nanostructure formation can be defined for an ablation rate less than several nanometers per pulse. For such a thin ablated layer, a uniform plasma surface is difficult to form. Here, let us define $F_{10 \mathrm{~nm}}$ as the fluence at which a $10-\mathrm{nm}$ layer is ablated. The values of $F_{10 \mathrm{~nm}}$ are listed in Table 1 . As shown in Fig. 2, the model is in fairly good agreement with the experimental results in the fluence range of $F_{10 \mathrm{~nm}}<F_{\mathrm{L}}<F_{\mathrm{M}}$, and a common fluence dependence of the periodic grating interspaces can be found for self-formation induced by femtosecond laser pulses for all metals. For Ti with relatively low melting temperature $\left(T_{\mathrm{m}}=1938 \mathrm{~K}\right)$ the grating nanostructures were self-formed for laser fluence of $0.09-$ $0.45 \mathrm{~J} / \mathrm{cm}^{2}$, while for Mo with high melting temperature $\left(T_{\mathrm{m}}=2888 \mathrm{~K}\right)$ structures were formed for laser fluence of $0.18-1.1 \mathrm{~J} / \mathrm{cm}^{2}$. Higher the melting temperature, upper
Table 1 Upper limit of grating structure formation $F_{\mathrm{M}}$, and $F_{10 \mathrm{~nm}}$ defined as the fluence at which a 10 -nm layer is ablated. $T_{\mathrm{m}}$ is the melting point.

\begin{tabular}{cccc}
\hline Material & $T_{\mathrm{m}}[\mathrm{K}]$ & $F_{\mathrm{m}}\left[\mathrm{Jcm}^{-2}\right]$ & $F_{10 \mathrm{~nm}}\left[\mathrm{Jcm}^{-2}\right.$ \\
\hline $\mathrm{Ti}$ & 1938 & 0.40 & 0.12 \\
$\mathrm{Pt}$ & 2042 & 0.44 & 0.24 \\
$\mathrm{Mo}$ & 2888 & 1.1 & 0.42 \\
$\mathrm{~W}$ & 3672 & 1.1 & 0.46 \\
\hline
\end{tabular}

limit of grating structure formation tends to be higher. These experimental results indicate that the period of the selfformed grating structure depend not on metal characteristics, but rather on the density of the surface plasma produced by a laser pulse. For high laser fluence $\left(F_{\mathrm{L}}>F_{\mathrm{M}}\right)$, the plasma expands too greatly for a clear surface to be constructed, or the plasma density is too high; in such cases, a surface plasma wave is not produced.

\section{Conclusions}

In summary, we measured the periodic structures selfformed on the surface of $\mathrm{Ti}, \mathrm{Pt}, \mathrm{Mo}$, and $\mathrm{W}$ metals. We found that the laser fluence dependence of grating structure period is the same for all metals on which the structure is self-formed. Higher the melting temperature of metals, upper limit of grating structure formation tends to be higher. The dependence can be explained by the induction of a surface plasma wave through the parametric decay of laser light, and can be reduced to the dependence of the density of laser-produced plasmas.

\section{Acknowledgments and Appendixes}

We thank Professors T. Kanaya, K. Nishida, and T. Terashima for their assistance with SEM and AFM. This research was financially supported by a Grant-in-Aid for the Global COE Program "The Next Generation of Physics, Spun from Universality and Emergence" from the Ministry of Education, Culture, Sports, Science and Technology (MEXT) of Japan and supported in part by the Amada Foundation for Metal Work Technology and a Grant-in Aid for Scientific Research (C)(22560720) from MEXT, Japan.

\section{References}

[1] K. Okamuro, M. Hashida, Y. Miyasaka, Y. Ikuta, S. Tokita, S. Sakabe, Phys. Rev. B 82 (2010) 165417.

[2] A. Y. Vorobyev, V. S. Makin, and C. Guo, J. Appl. Phys. 101 (2007) 034903.

[3] A. Y. Vorobyev and C. Guo, Appl. Surf. Sci. 253 (2007) 7272-7280.

[4] M. Tsukamoto, K. Asuka, H. Nakano, M. Hashida, M. Katto, N. Abe, and M. Fujita, Vacuum 80 (2006) 1346-1350.

[5] V. S. Makin, R. S. Markin, A. Ya Vorobyev, and C. Guo, Tech. Phys. Lett. 34 (2008) 387-390.

[6] A. Y. Vorobyev, and C. Guo, J. Appl. Phys. 104 (2008) 063523.

[7] Q. Z. Zhao, S. Malzer, and L J. Wang, Opt. Lett. 32 (2007) 1932-1934.

[8] E. V. Golosov, V. I. Emel'yanov, A. A. Ionin, Yu. R. Kolobov, S. I. Kudryashov, A. E. Ligachev, Yu. N. 
Novoselov, L. V. Seleznev, and D. V. Sinitsyn, JETP Lett. 90 (2009) 107-110.

[9] J. Wang and C. Guo, J. Appl. Phys. 100 (2006) 023511.

[10] A. Y. Vorobyev, and C. Guo, J. Appl. Phys. 103 (2008) 043513.

[11] J. Wang and C. Guo, Appl. Phys. Lett. 87 (2005) 251914.

[12] J. Gottmann, D. Wortmann, and M. H-Jungemann, Appl. Surf. Sci. 255 (2009) 5641-5646.

[13]A. Y. Vorobyev, and C. Guo, Phys. Rev. B 72 (2005) 195422.

[14] J. Kim, S. Na, S. Cho, W. Chang, and K. Whang, Opt. Lasers Eng. 46 (2008) 306-310.

[15] S. E. Kirkwood, A. C. Popta, Y. Y. Tsui, R. Fedosejevs, Appl. Phys. A 81 (2005) 729-735.

[16] A. Weck, T. H. R. Crawford, D. S. Wilkinson, H. K. Haugen, and J. S. Preston, Appl. Phys. A 89 (2007) 1001-1003.

[17] A. Weck, T. H. R. Crawford, D. S. Wilkinson, H. K. Haugen, and J. S. Preston, Appl. Phys. A 90 (2008) 537-543.

[18]R. V Volkov, D. M. Golishnikov, V. M. Gordienko, and A. B. Savel'ev, JETP Lett. 77 (2003) 473-476.

[19] A. Y. Vorobyev and C. Guo, Appl. Phys. A 86 (2007) 321-324.

[20]B. K. Nayak, M. C. Gupta, and K. W. Kolasinski, Appl. Phys. A 90 (2008) 399-402.

[21] V. Oliveira, S. Ausset, and R. Vilar, Appl. Surf. Sci. 255(2009) 7556-7560.
[22] Y. Huang, S. Liu, W. Li, Y. Liu, and W. Yang, Opt. Express 17 (2009) 20756-20761.

[23] Q. Z. Zhao, S. Malzer, and L.J. Wang, Optics Express 15 (2007) 15741-15746.

[24] M. Hashida, K. Nagashima, M. Fujita, M. Tsukamoto, M. Katto, and Y. Izawa, Proceedings of 9th Symposium on Microjoining and Assembly Technology in Electronocs, edited by K. Atsumi (Japan Welding Society, Tokyo, 2003), Vol. 9, pp. 517-522.

[25] S. Sakabe, M. Hashida, S. Tokita, S. Namba, and K. Okamuro, Phys. Rev. B 79 (2009) 033409.

[26] S. Juodkazis, A. V. Rode, E. G. Gamaly, S. Matsuo, H. Misawa, Appl. Phys. B 77(2003)361-368.

[27] M. Hashida, S. Namba, K. Okamuro, S. Tokita, and S. Sakabe, Phys. Rev. B 81(2010) 115442.

[28] E. G. Gamaly, Phys. Rep. 508(2011)91-243.

[29] M. Hashida, Y. Miysakasa, Y. Ikuta, S. Tokita, and S. Sakabe, Phys. Rev. B 83(2011)235413.

[30] R. Buividas, L. Rosa, R. Sliupas, T. Kudrius, G. Slekys, V. Datsyuk and S. Juodkazis, Nanotechnology 22(2011)055304.

[31] Y. Shimotsuma, P. G. Kazansky, J. Qui, and K. Hirao, Phys. Rev. Lett. 91 (2003) 247405.

[32] G. Miyaji and K. Miyazaki, Opt. Express 16 (2008) 16265-16271.

[33] J. Reif, O. Varlamova, and F. Costache, Appl. Phys. A 92 (2008) 1019-1024.

[34] S. Tokita, M. Hashida, S. Masuno, S. Namba, and S. Sakabe, Opt. Express 16(2008)14875.

(Received: July 14, 2011, Accepted: April 12, 2012) 